\title{
A New Paradigm of Pharmaceutical Drug Delivery Systems (DDS): Challenges for Space, Time, and Shapes
}

Jaeseok Eo ${ }^{1}$, Brandon Cepeda ${ }^{1}$, Jihye Kim², and Namsoo Kim, PhD ${ }^{1,2}$

${ }^{1}$ Department of Metallurgical, Materials and Biomedical Engineering, The University of Texas at EI Paso, TX, USA

${ }^{2}$ Center for Printable Materials Certification (CPMC), The University of Texas at El Paso, TX, USA

\begin{abstract}
Using 3D food printing with the Internet of Things (IOT) technology, patients can receive diagnoses and prescriptions from their doctors while in the comfort of their homes. The patient-specific prescription has been innovated by converging 3D food printing technology with drug delivery systems (DDSs). Quantitative drug dosages can be incorporated into the composition of food and produced in any shape within a short time. Automating food and DDSs makes promising implications for healing patients remotely, as well. Each of these aspects, along with IOT technology, have contributed to increased health care for patients, no matter their location. The quantitative discharge of vitamin C melted in water, mayonnaise, ketchup, and peanut butter has been verified using the Piston Typed Extrusion (PTE) method. Designs with different curves and shapes were repeatedly printed with a head speed of $1.6 \times 10^{-2} \mathrm{~m} / \mathrm{s}$, and it was confirmed that effective control while printing the shapes was possible. The Hagen-Poiseuille (HP) formula was utilized to simulate the overall printing time. This simulation affirmed that increasing the head speed from $1.6 \times 10^{-2} \mathrm{~m} / \mathrm{s}$ to $4.0 \times 10^{-2} \mathrm{~m} / \mathrm{s}$ had reduced the printing time consistently, but the time was not reduced continuously after $4.0 \times 10^{-2} \mathrm{~m} / \mathrm{s}$, depending on the materials' viscosities and how much curvature exists in the designs. The precision of printing was adjusted within $5 \%$ of the theoretical value during printing, and the loT technology allowed printing of the materials within five minutes, regardless of the patient's location.
\end{abstract}

Keywords: Drug Delivery System (DDS); Internet of Things (IOT); Quantitative Control; 3D Printing; Patient-Customized; Piston Type Extrusion (PTE)

\section{Introduction}

The historical Greek physician Hippocrates said no medicine is needed since there is no illness that cannot be cured with food. ${ }^{1}$ In the modern age, the advantages of food and medicine have been placed hand-in-hand for the benefit of humanity. A revolutionary drug delivery system (DDS) that accurately controls the dosages of drugs within food's composition has been studied. The development of a DDS to transmit precise amounts of drugs remotely has been a long-term interest in the medical field. DDSs have focused on a continuous delivery mechanism within the body by the use of microstructures in materials, and this field has been researched. ${ }^{2,3,4}$ One example of these mechanisms is the Ringer's solution. As the most preferred technique for drug transmissions, a Ringer's solution is given to people who feel fatigued. All of the solutions appear transparent, but there are hundreds of species, depending on its purpose. Basic solutions are prescribed to replenish the water levels of patients whose electrolytes are not balanced due to possible difficulties in drinking water or abnormalities in their heart or kidneys. Nutritional solutions are mixtures of solutions. Proteins, carbohydrates, fats, vitamins, and trace elements can be supplemented through these solutions.

Corresponding author: Namsoo Kim, PhD

The University of Texas at EI Paso, TX, USA

Tel: +1 915-747-7996; fax: +1 915-747-8036;

E-mail: nkim@utep.edu
Special solutions are used for particular medical situations. ${ }^{5}$ In cases of blood infusions, precise amounts of dosages can be administered to a patient, depending on their age, weight, and clinical or biological conditions. ${ }^{6}$ These scenarios include administering a solution that lowers the pressure being placed on a patient's brain due to cerebral hemorrhaging and dispensing a solution to keep the amount of blood flow constant.

The technique of making the solutions has contributed to the development of macro-DDSs by shining light on how versatile DDSs can become. This versatility has been achieved with basic solutions, nutritional solutions, and special solutions, but the process of making these solutions is not easy, and many errors can occur. ${ }^{5}$ It carries the risk of underdosing or overdosing patients, and medical doctors or nurses are incapable of being ever present to monitor each patient for these risk factors. The equipment involved in this method can be cumbersome. ${ }^{6}$ When a macro-DDS is applied, the solutions can be accurately delivered to the patient from remote locations, and customized medication can be created for each patient using the Internet of Things (IOT) technology. ${ }^{7}$ The new field of macro-DDSs will span beyond the confines of micro-DDSs using loT-based technology. This novel application has the potential to be directly involved in disaster situations or in health care to deal with daily food management for consumers without space constraints. Future DDSs are based on this recent concept as it can be utilized in transferring precise amounts of drugs without space constraints, mixing viscous substances, 
solidifying a fluid for consumption when a patient may not be able to ingest liquids, and using equipment that quantifies the liquid state of a solution onto the surface of solidified food. Implementation of macro-DDS will lead to a new health technique for quantitative medicinal management.

Production of quantified medication by this unique pharmaceutical system requires 3D printing technology for real practice. The applicability of 3D printing technology in the medical field has already been extensively explored. One instance is that of the research conducted on microneedle patches. ${ }^{8}$ Microneedle patches are dime-sized plastic strips that contain tens to hundreds of drug-filled microscopic needles. These needles are soluble in water, and little to no pain is felt when applied to a patient's skin. ${ }^{9}$ Another example of 3D printing in the medical field is the use of UV light to manufacture drug-containing structures by crosslinking. These structures, with different pore sizes and different drug loadings, were able to control drug release rates using $3 \mathrm{D}$ printing technology. ${ }^{10}$

What each of these medical devices have in common is that they are produced using 3D printing technology. Challenges dealing with space, time, and shapes remain with these innovative DDSs. Effective drug delivery must not only be quantitatively controlled, but also must be free from space constraints, delivered in a short time, and printed with patientpreferred shapes in order to be easily accessed by the patients. The authors of this study have submitted a patent on this DDS, and it is currently being utilized for pharmaceutical trainings and education. ${ }^{11}$ The trainees have succeeded in simulating 3D printing with the quantitative ejection of nutrients and carbohydrates into food. ${ }^{12}$ This research is encouraging since it shows that, if high viscosity food material can be quantifiably printed, high viscosity drug solutions can be as well. This technology has been continuously tested since 2018 in the joint degree program between The College of Engineering and The School of Pharmacy. ${ }^{12}$

Advancements in 3D printing technology enabled custom applications to produce personalized drug treatments for individuals via the use of both the Cloud and robotic technologies. ${ }^{13,14}$ For innovative DDS to be practical, space constraints must be overcome through the loT technology system. To illustrate the loT process, it can be divided into four sections: the Cloud, where information is accessed; MongoDB, the database where information is stored; Raspberry Pi (RP), the microprocessor of the system; and Arduino, the microcontroller of the printer. The cloud displays printing conditions, printing designs, user data, locations, and codes for available printers in a database called MongoDB. ${ }^{15}$ When a printer or a user requests any data, the stored data from MongoDB is transferred to the RP. The RP of the printer receives the data and orders the Arduino board to control the movement of the printer head according to its printing conditions and design. RP microprocessing equipment, with an Arduino microcontroller, has been patented. ${ }^{7}$ A patient's health information is entered through a smartphone device. Then, this data is stored and analyzed by MongoDB in the Cloud. This data can be recalled to smartphone devices at any time. MongoDB calculates food printing conditions based on the analyzed data. The user selects the design and requests for it to be printed. Next, the saved G-code file is transferred to the RP of the printer. When an RP sends orders to an Arduino board, printing begins to produce food that fits the health conditions of patients. Because all the data is stored in the cloud, it can be retrieved and printed from anywhere whenever the device and printer are connected to the Wi-Fi.

By finding practical ranges with real-time analysis of weight, head movement, temperature, etc, the optimal printing conditions have been thoroughly examined. First, data was sent to the Cloud from the RP. Next, the data was analyzed and sent back to the RP board from the Cloud server. These signals were transmitted from the RP to an Arduino board to initiate the printing operation. ${ }^{16}$ Applications on the Cloud can access microcontroller-based devices and collect data from them. This is what is meant by the term Internet of Things. ${ }^{16}$ The "IOTenabled 3D Food Printer" had been successfully used by pharmacists, patients, and their family members through the food control program of the pharmacy. ${ }^{7,12}$

With loT technology, being able to 3D print drugs onto food from a distance with accurately customized ingredients can be achieved. By incorporating protein sources, healthier diet options for patients who have certain restrictions on what they can consume can be created. ${ }^{17}$ Specialists can properly prescribe what the patients or elderly would need based on previously gathered data from a remote location. Specialists can also determine which foods or nutrients need to be added to patients' diets by using high viscosity material extrusion technology. The assistants attending to the patients or elderly can use their smart devices to have the patient's food printed, or get the food or drugs sent to the patient right when they are ready without the patient having to prepare anything else. After the prescription is given to the patient and feedback is received from them, loT technology can store the data and analyze the exact amount of each ingredient or drug that needs to be prescribed next time. Accumulation of prescription data and printed conditions will decrease more and more errors during printing or drug delivery with respect to time.

Removing space constraints is essential in ensuring that patients globally can receive adequate healthcare using loT technology. The loT technology allowed DDSs to become remote and adaptable to different situations. Automating food and DDSs for disaster relief efforts is a possible 
implementation of this technology, since raw ingredients can be shipped to remote areas easily. This means, as machines handle the preparation of food, medical responders can focus on treating the injured or ill. Infrastructure for monitoring consumers' medical data can also be established, allowing the assessment of changes in health conditions in real-time. These features, in combination with loT technology, will be essential in improving patients' health.

The applicability of IoT technology has previously been demonstrated through preliminary testing. Using twenty donated 3D printers in cooperation with a company based in South Korea, the research team at The University of Texas at El Paso (UTEP) achieved in video conferencing employees of the company while simultaneously having products 3D printed at the company using loT based 3D printers. Represented in Fig. 1 is the conceptual diagram of loT technology being employed. In this study, remote printing was successfully conducted by sending a design file from the Engineering building at UTEP to The School of Pharmacy located $1.61 \mathrm{~km}$ away with a smartphone.

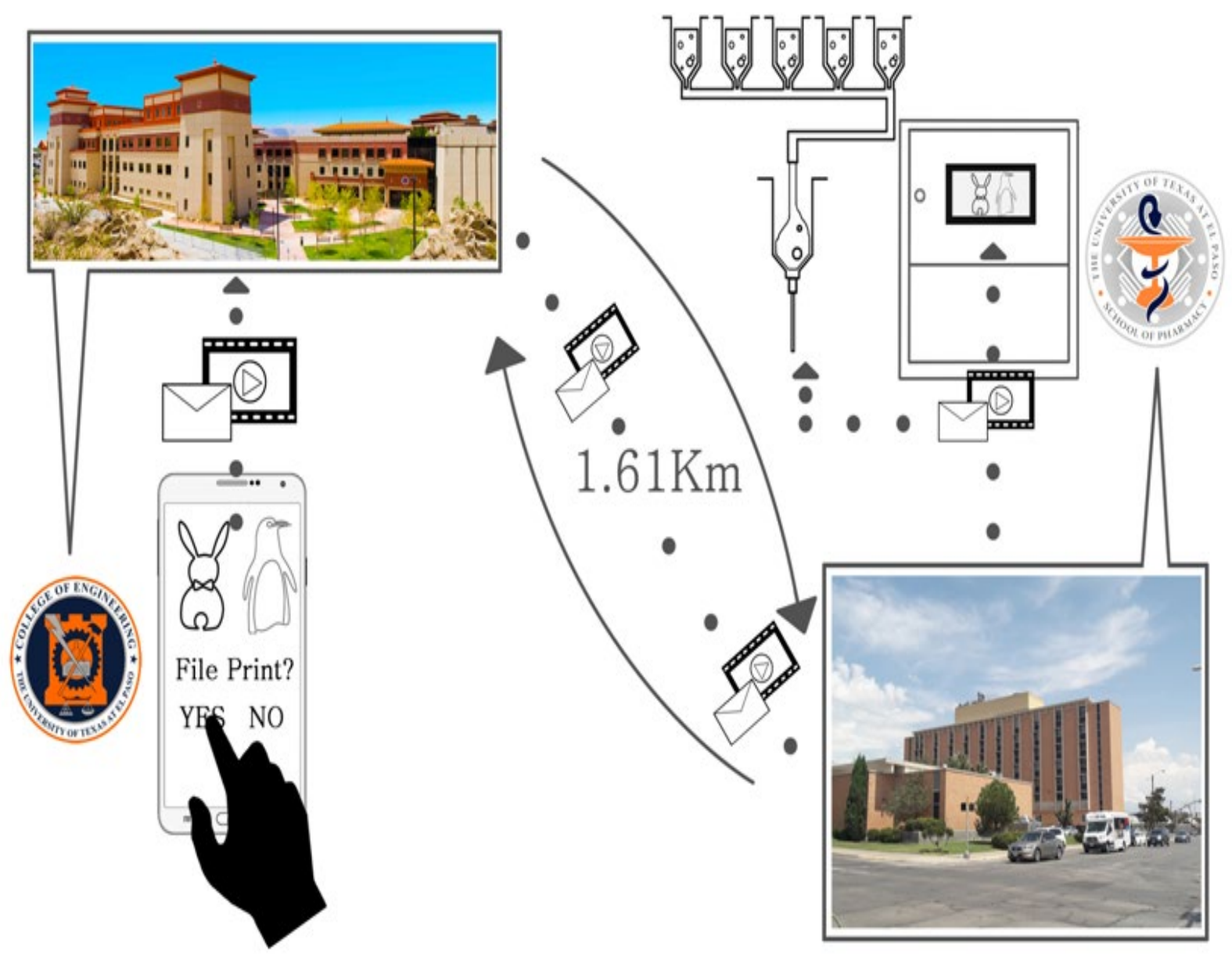

Figure 1. Conceptual diagram of using loT.

The study aimed to improve the potential of quantitative drug delivery by $3 \mathrm{D}$ printing with food and changing the delivered drug amounts based on the printed design or shape. Using 3D printing techniques rather than ordinary ingestion or injection of the drug is expected to reduce the errors of drug delivery. The 3D printer's capability to control the flow rate or printing speed can allow quantitative drug control on any design or shape. ${ }^{18}$ In order to confirm this hypothesis, the optimization of time and accuracy of 3D printing drugs with food, the quantitative discharge of the material, and evaluation of the potential of the new DDS in conjunction with the loT system has been observed. 


\section{Experimental Methods}

Equipment

The PTE method allowed the quantitative printing of highly viscous food material or biomaterial. ${ }^{16,19-22}$ The designs of the developed loT-enabled equipment were patented and used for testing in this research. ${ }^{7,23,24}$
Using the printers, medication was printed into food products or onto the food's surface. The combination of the food and medical industries has allowed the customization of food with specific amounts of ingredients and drug dosages for patients in the manner that was previously described.

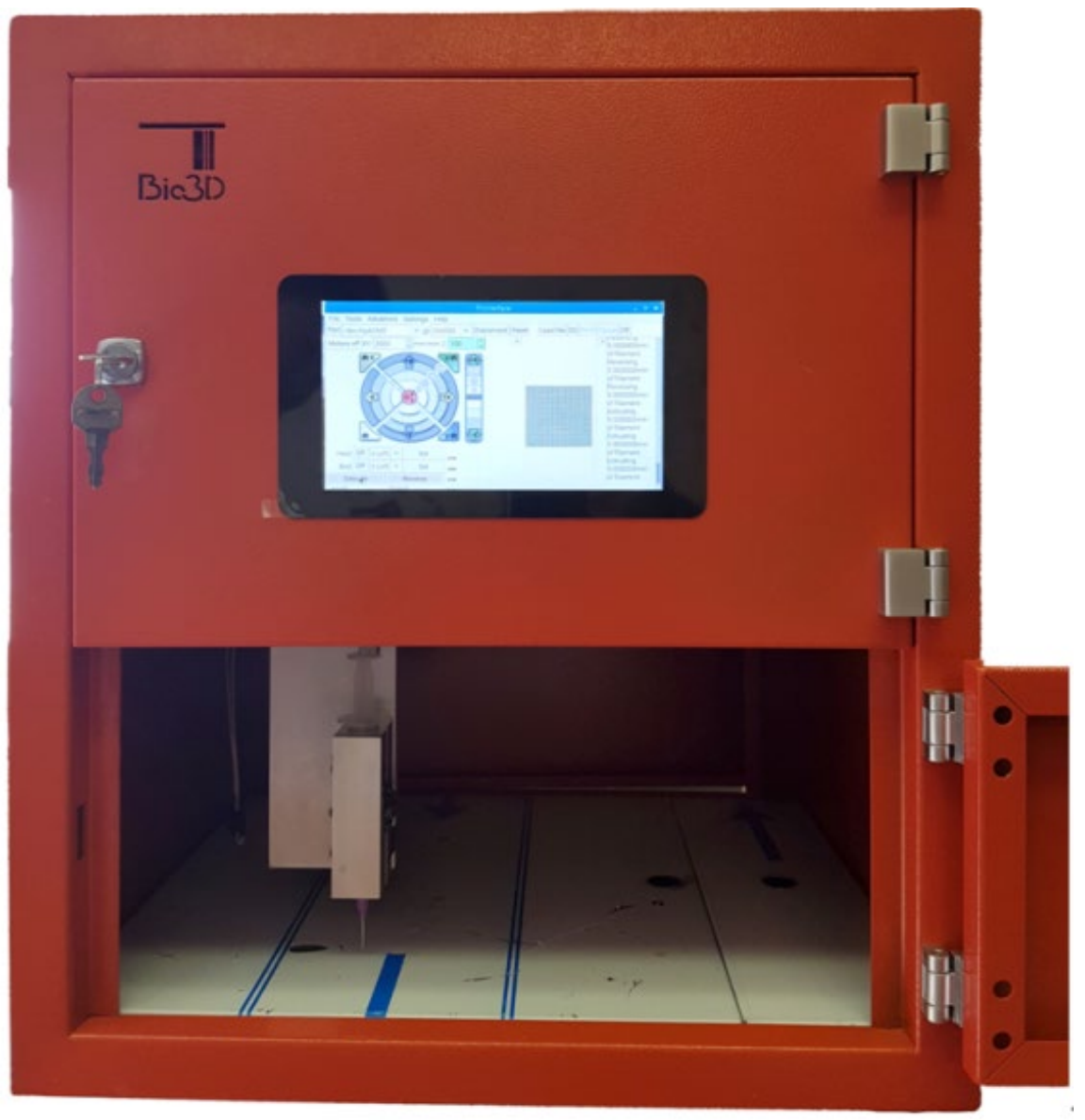

Figure 2. The loT-enabled 3D printer. ${ }^{24}$

\section{Materials}

In the experiment, water, mayonnaise, ketchup, and peanut butter, which are all highly viscous victuals, were used to verify that the needed components were dissolved in a solution and that the composition was controlled. Any nutrients and their compositions can be controlled and placed in a variety of highly viscous liquids in the form of a Ringer's solution, allowing patients to receive more accurate amounts of nutrients from remote locations. Represented in Table 1 is the viscosity, density, pressure, and flux of each of the used materials. 
Table 1. Conditions of used materials extruded using a tip-diameter of $1.66 \times 10^{-3} \mathrm{~m}$ and tip-length of $2.30 \times 10^{-2} \mathrm{~m}$.

\begin{tabular}{|c|c|c|c|c|}
\hline Materials & $\begin{array}{c}\text { Viscosity } \\
(\mathrm{Pa} \cdot \mathrm{s})\end{array}$ & Density $\left(\mathrm{g} / \mathrm{m}^{3}\right)$ & Pressure (MPa) & $\begin{array}{c}\text { Flux: } \mathrm{q}_{\mathrm{w} \times 10^{2}} \\
(\mathrm{~g} / \mathrm{s})\end{array}$ \\
\hline Water (10wt.\% medicine) & $0.001-10^{*}$ & $1.10 \times 10^{6}$ & 0.101 & $3.74 \pm 0.23$ \\
\hline Mayonnaise* (10wt.\% medicine) & $5-100^{*}$ & $1.03 \times 10^{6}$ & 0.123 & $3.77 \pm 0.28$ \\
\hline Ketchup* (10wt.\% medicine) & $75-100$ & $9.03 \times 10^{5}$ & 0.432 & $3.60 \pm 0.22$ \\
\hline Peanut Butter* (10wt.\% medicine) & $250-1000$ & $1.06 \times 10^{6}$ & 1.205 & $3.53 \pm 0.33$ \\
\hline
\end{tabular}

*Viscosity of material varies with temperature and compositions

\section{Quantitative Control}

The most important feature of loT-enabled 3D printing for patients in the medical field is quantitative control of the material when being discharged. Since any minimal changes in the amount or composition of drugs or food may worsen diseases or cause severe side effects for patients and the elderly, IoT technology will contribute significantly in easing symptoms and reducing side effects. This technology features scales that allow operators to control and verify how much of a material is discharged during printing. To ensure the applicability of this technology in a Ringer's solution, a supply of medical salt water had been discharged within the solution. Whereas the injection error range in a typical injection method is between 5 and $10 \mathrm{ml}$ based on $150 \mathrm{ml}$, salt water was experimented with to find the range or pressure signal where the more accurate injection was possible. The experiment was conducted by injecting salt water into distilled water, then injecting various materials repeatedly within the solution.

Time and Accuracy with Shape of Printing

Drugs manufactured using loT technology in the field should be delivered to the patients as soon as possible. A certain amount of medicine must be printed within three minutes and accurately delivered to where it is needed. Designs with different curves and shapes were printed with a head speed of $1.6 \times 10^{-2} \mathrm{~m} / \mathrm{s}$, and a vitamin C composition of $10 \mathrm{wt} . \%$ was melted in ketchup. The experiment was repeated to effectively print the entire drug by controlling the high curvature and size of the shapes using different designs. The theoretical error range and accuracy of printing was obtained by calculating the difference in volume between each discharged print.
Printing Stability in Relation to Head Traveling Speed

The stability of the printed shape, with respect to increasing head speed, was observed. Since drugs produced using IoT technology must be delivered in a short time, an attempt was made to print a certain amount of a drug within 10 minutes, then accurately deliver it. For this purpose, how accurately the different shapes were printed with the slowest speed of $2.0 \times 10$ ${ }^{2} \mathrm{~m} / \mathrm{s}$ needed to be known. When printing the various shapes, ketchup and mayonnaise were used as ingredients with vitamin $\mathrm{C}$ incorporated into them. The HP formula was also utilized to simulate the overall printing time. This assisted in reducing the printing time by increasing the head traveling speed and predicting the accuracy and stability of discharge of the materials.

\section{Results and Discussion}

Time and Accuracy with Shape of Printing

In the case of printing vitamin $C$ into the ketchup with a head speed of $1.6 \times 10^{-2} \mathrm{~m} / \mathrm{s}$; it was confirmed that the vitamin was evenly distributed within $1.8 \%$ from the target position, regardless of the shape used. Provided in Table 2 below are the printed shapes, and the absolute amounts of vitamin $C$ added to them by experimenting with the given shapes repeatedly with the head speed of $1.6 \times 10^{-2} \mathrm{~m} / \mathrm{s}$. The PTE method was used, along with a tip diameter of $1.6 \times 10^{-3} \mathrm{~m}$. Depending on the shape shown in the table below, it was found that $101.5 \mathrm{mg}$ to $376.4 \mathrm{mg}$ of the vitamin was printed, which took between 27.5 seconds to 102 seconds to print. 
Table 2. Time consumption and vitamin Cloaded capacity in each printed design. The line of printing time by total weight indicated that all of the materials were quantitatively discharged for 300 seconds using a tip-diameter $1.5 \times 10^{-3} \mathrm{~m}$ and tip-length $1.80 \times 10^{-2} \mathrm{~m}$.

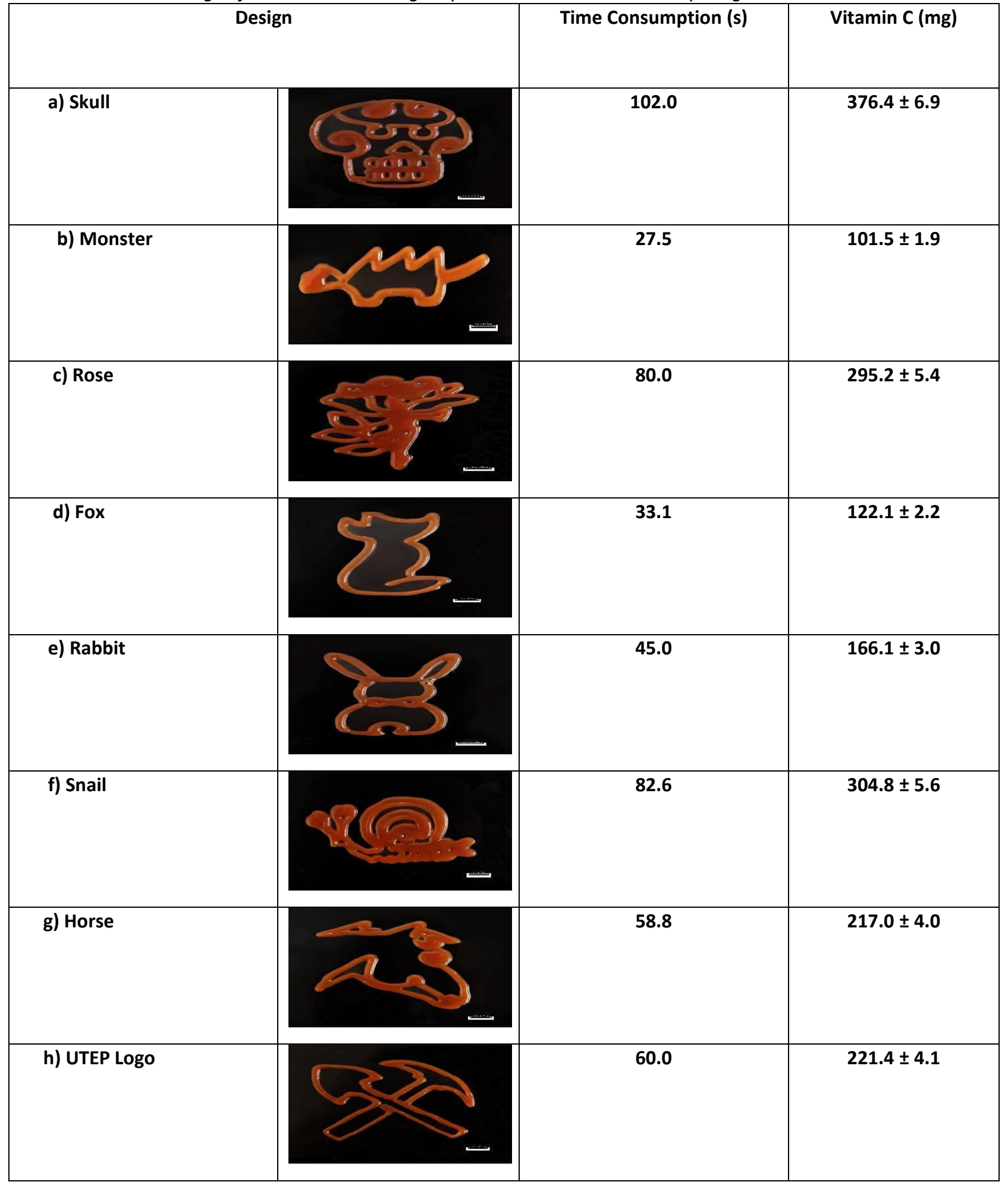




\section{Time Savings}

An effect of increasing the head speed on the printing time and the stability of the printed shape was observed. Shown in Fig. 3 are the times taken to print each of the shapes by increasing the head speed relative to the time taken at $2.0 \times 10^{-2} \mathrm{~m} / \mathrm{s}$. Each shape's time varied depending on its curvatures and angular parts. These shapes were printed by increasing the head speed from $2.0 \times 10^{-2} \mathrm{~m} / \mathrm{s}$, which takes about 30 minutes, to confirm the point of highest stability in the figure where quantitative discharge was possible. It was confirmed that any shape could be stably printed in the range of $4.0 \times 10^{-2} \mathrm{~m} / \mathrm{s}$ to $4.8 \times 10^{-2} \mathrm{~m} / \mathrm{s}$. In the case of complex shapes, increasing the head speed over $6.0 \times 10^{-2} \mathrm{~m} / \mathrm{s}$ did not reduce the time needed. As the curvatures or bending in the shape increases, so does the amount of time needed to print it.

Time Reduction and Accuracy by Head Traveling Speed

By incrementally increasing the head's speed by $0.8 \times 10^{-2} \mathrm{~m} / \mathrm{s}$ from $1.6 \times 10^{-2} \mathrm{~m} / \mathrm{s}$, regardless of the printed shape, the time taken to complete each print was divided by the printing time at the head speed of $1.6 \times 10^{-2} \mathrm{~m} / \mathrm{s}$. This value is described as the Printing Time Ratio in the figure below. If the head speed is increased by a factor of two, the Printing Time Ratio should be halved. The reduction of time, according to the HP formula, is represented by a dotted line and labeled as the Ideal Case. It was confirmed through the simulation that increasing the head speed does not reduce the printing time continuously, depending on different viscosities of materials and how much bending is prevalent in the designs. The simulation results confirmed that, if the head speed was increased from $1.6 \times 10^{-2}$ $\mathrm{m} / \mathrm{s}$ to $4.0 \times 10^{-2} \mathrm{~m} / \mathrm{s}$, the printing times did not deviate significantly from the theoretical value, regardless of the shape. It was shown from the printing simulation below that the time required for actual printing was not significantly reduced, unlike the theoretical prediction. This depended on stopping of the material, bending, changes of direction, etc.

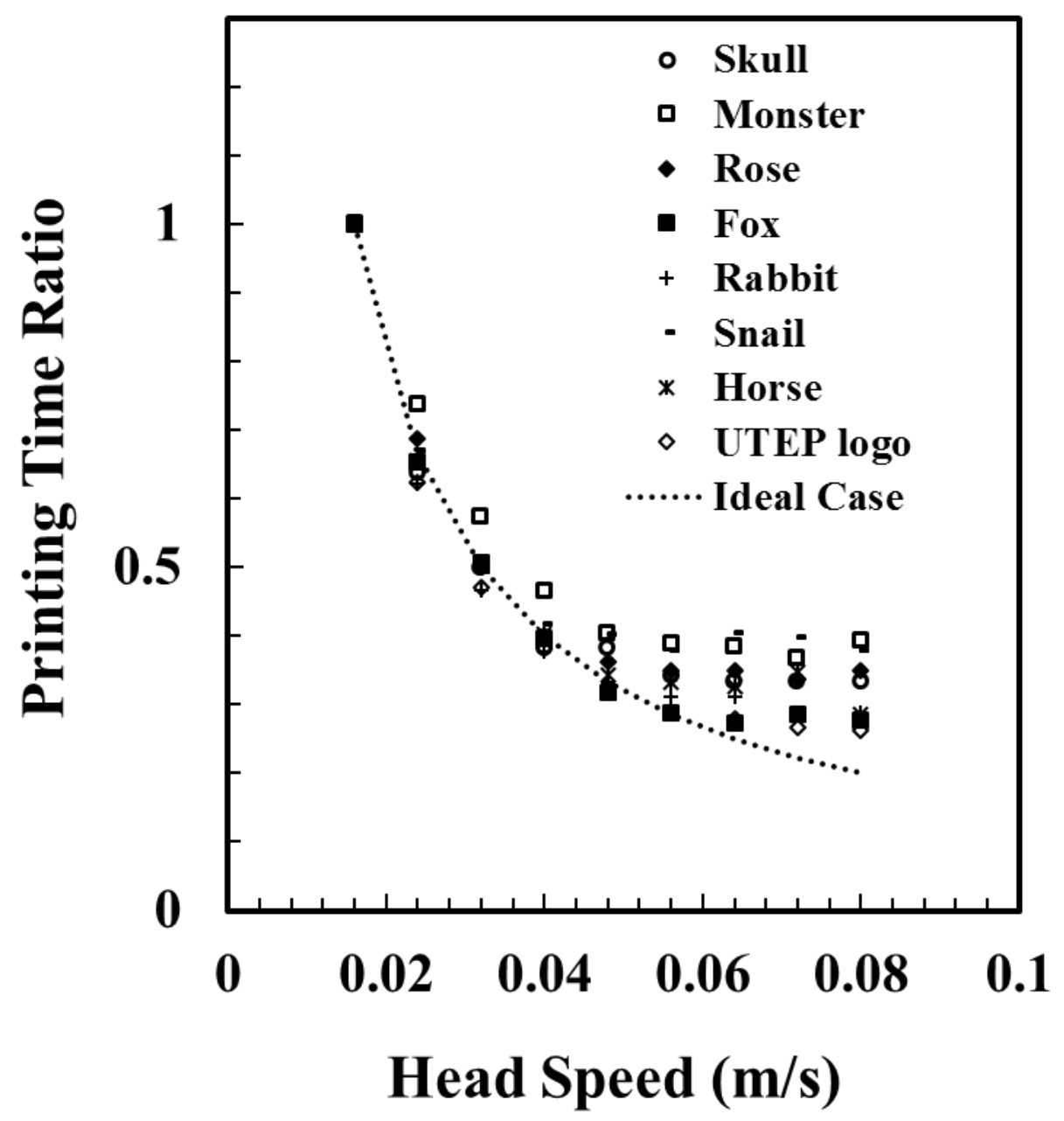

Figure 3. Printing time in relation to printing head speed by design and shape of the eight models. 
As shown in the results in Fig. 4 and Fig. 5, the head speed was increased by intervals of $0.08 \mathrm{~m} / \mathrm{s}$ from $1.6 \times 10^{-2} \mathrm{~m} / \mathrm{s}$ to $4.0 \times 10$

$-2 \mathrm{~m} / \mathrm{s}$ while producing a penguin design. As a result of printing the shape repeatedly, the total discharge with increasing head speed was $1.80 \pm 0.05 \mathrm{~g}$ and the accuracy percentage was $2.96 \%$, in the case of ketchup. For the mayonnaise with vitamin $\mathrm{C}$ added, the total discharge was $1.49 \pm 0.06 \mathrm{~g}$ with $4.22 \%$ accuracy.

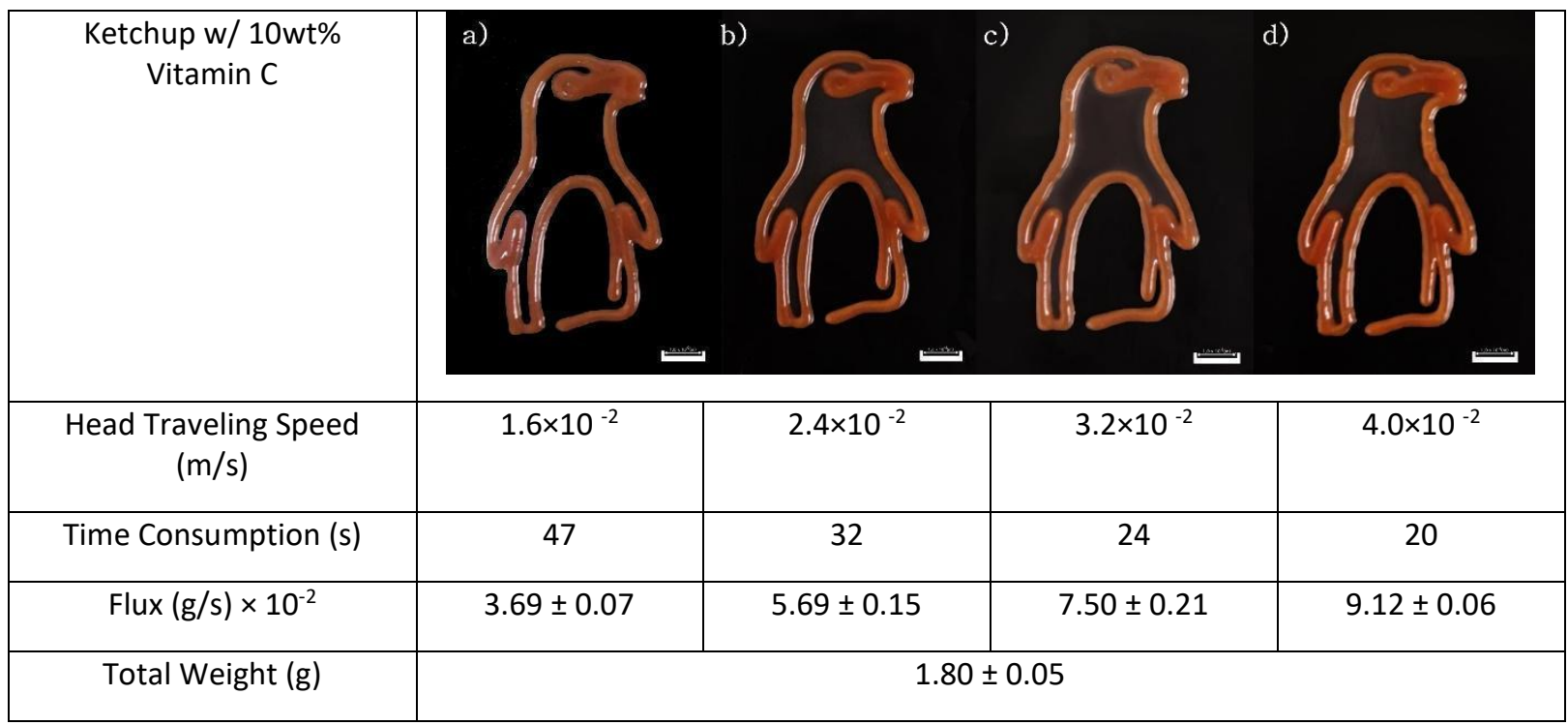

Figure 4. Printing time reduction and accuracy by printing speed with vitamin $C$ loaded ketchup using a tip-diameter of $1.5 \times 10^{-3} \mathrm{~m}$ and tip-length of $1.80 \times 10^{-2} \mathrm{~m}$.

As shown in Fig.4 and Fig. 5, when the head speed was $4.0 \times 10^{-}$ ${ }^{2} \mathrm{~m} / \mathrm{s}$, the discharge strength outrunning the friction between the material and the piston tip was constant. The error range was $0.68 \%$ for ketchup and $2.6 \%$ for mayonnaise, which were lower than that of the average error range. As presented in Fig.
4 and Fig. 5, the stability of the shape was low when it was printed using a head speed of $4.0 \times 10^{-2} \mathrm{~m} / \mathrm{s}$. The surfaces of the printed materials were not smooth, showing incomplete shapes compared to other head speed conditions. The roughness on the surface aggravated as the shape became more complicated.

\begin{tabular}{|c|c|c|c|c|}
\hline $\begin{array}{c}\text { Mayonnaise w/ 10wt\% } \\
\text { Vitamin C }\end{array}$ & a) & $4.0 \times 10^{-2}$ \\
\hline Head Traveling Speed \\
(m/s)
\end{tabular}

Figure 5. Printing time reduction and accuracy by printing speed with vitamin $C$ loaded mayonnaise using a tip-diameter of $1.5 \times 10^{-3} \mathrm{~m}$ and tip-length of $1.80 \times 10^{-2} \mathrm{~m}$. 
Quantitative Discharge with Respect to Time

Provided in Fig. 6 below are the analyzed weights of the printed designs, according to the printing time. Using the PTE method, (a) water, (b) mayonnaise, (c) ketchup, and (d) peanut butter were printed with $500 \mathrm{mg}$ of the drug once it was melted. As displayed in the graph of Fig. 4, the proportional line of the printing time by the total weight indicated that all of the materials were quantitatively discharged for 300 seconds. In order to decrease the change in pressure $(\Delta P)$ and optimize the extrusion, a tip-diameter of $1.66 \times 10^{-3} \mathrm{~m}$ and tip-length of $2.30 \times$ $10^{-2} \mathrm{~m}$ was used. $10 \mathrm{wt} . \%$ of vitamin $\mathrm{C}$ was included in the food material, lowering its viscosity. This has increased $\Delta P$ within the piston, increased the flux of the materials $\left(q_{w}\right)$, and increased its total discharge weight for all experiments from the expected or theoretical value.
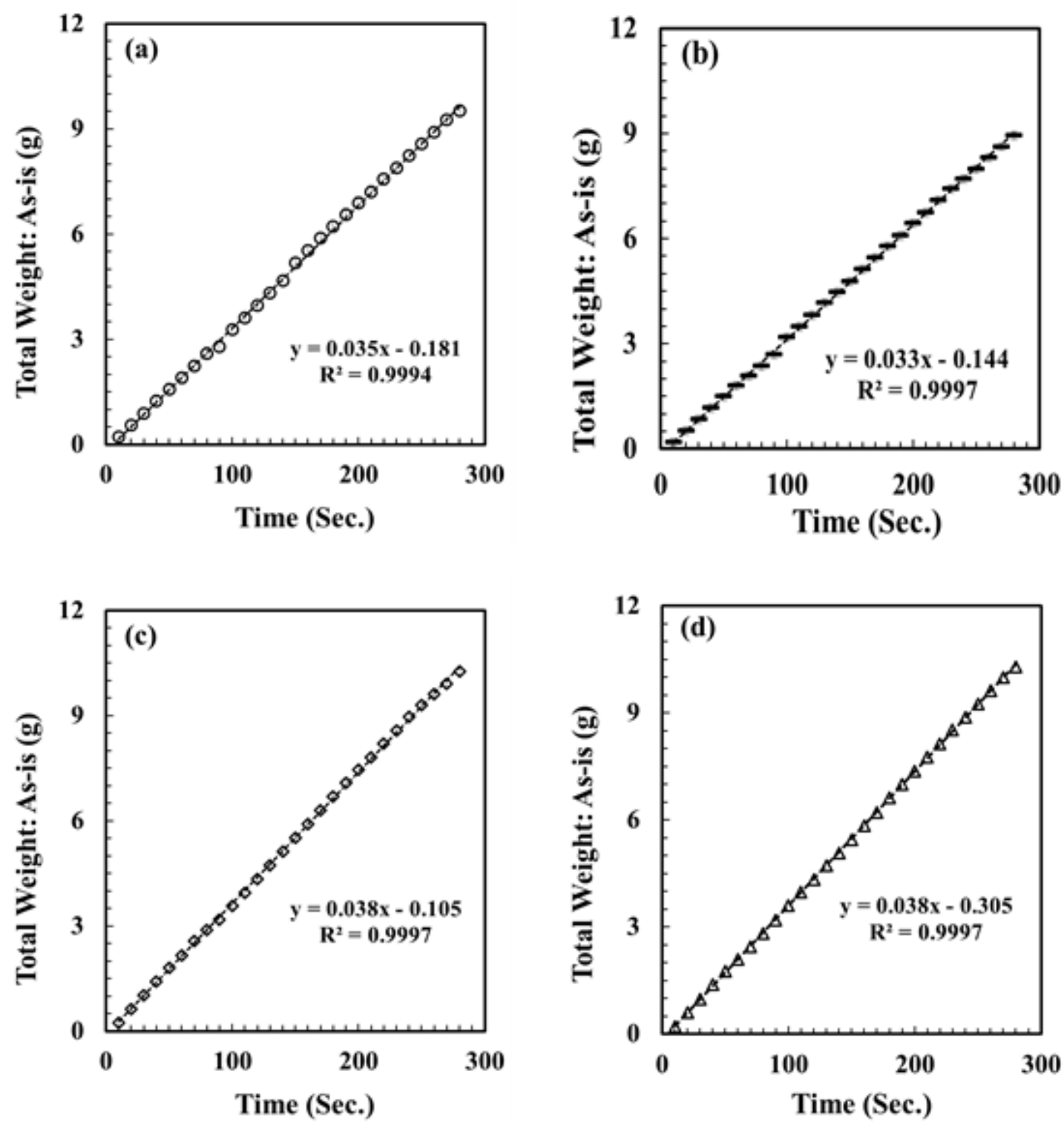

Figure 6. Measured weight of each printed design using (a) water, (b) mayonnaise, (c) ketchup, and (d) peanut butter printed with $10 \mathrm{wt} . \%$ of the drug. 
As confirmed by the previous experiment, where the results are shown in Fig. 6, quantitative control of the materials during discharge was attained. The outcome of the research is promising since it manifests future uses in controlling the exact amount of any nutrient varying from carbohydrates to fats and medication, allowing for the customization of patients' diets. As displayed in Fig. 7, the patients are capable of adding or removing their prescribed medications or nutrients from their preference of food. Previous DDSs have been based on targeting and control methods that are not regularly attainable for patients unless they are present at a medical facility. ${ }^{25-27}$ Rather than reusing these standard DDS methods, controlling the composition of the food and medication, as shown below, will result in increased accessibility for patients who have difficulties with mobility.

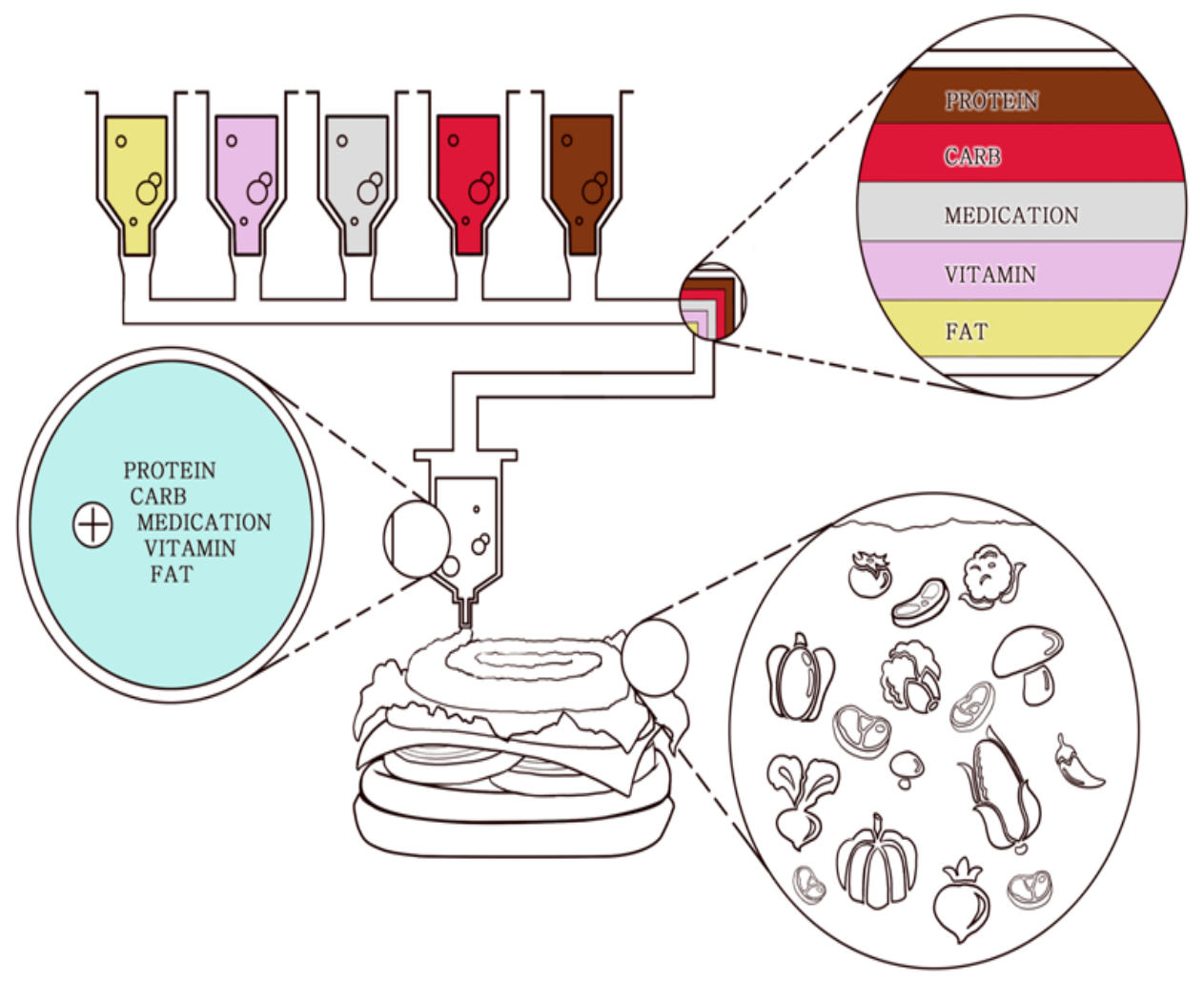

Figure 7. Controlled printing of the nutrients or medication incorporated into different food products.

\section{Patient-specific Drugs}

For many medications, drug intake is highly recommended during meals, 30 minutes after meals, or immediately after meals. In the case of those living with diabetes, patients must intake the drugs within 30 minutes after a meal for the drugs to be effective. However, there is the problem of the elderly or children forgetting to take their prescribed medication, which can cause side effects or symptoms to intensify.

Making medication in the form of liquid solutions and pills enabled elderly and ill patients to effectively intake drugs by mixing them with ordinary food. Exhibited in Fig. 8 are printed products with various shapes and materials. This showed that the drugs can be printed in any form or shape on the food. Assuming that 500 to $1000 \mathrm{mg}$ of Metformin $\mathrm{HCl}$ was ingested, if the solution was produced with a high viscosity material by dispersing it in an aqueous solution, the addition of chemicals, water, and other solids would allow the adjustability of the drug content to $10 \mathrm{wt} . \%$ of the fluid. The patient may intake a $10 \mathrm{wt} \%$ Metformin slurry in water 5 to $10 \mathrm{~g}$ at a time. It was confirmed that the drug can be delivered in various shapes by printing less than $10 \mathrm{~g}$ onto food, such as semi-solid water, mayonnaise, ketchup, jam, and peanut butter. 
c)

a)

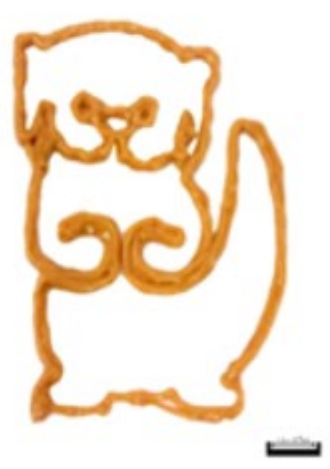

d)

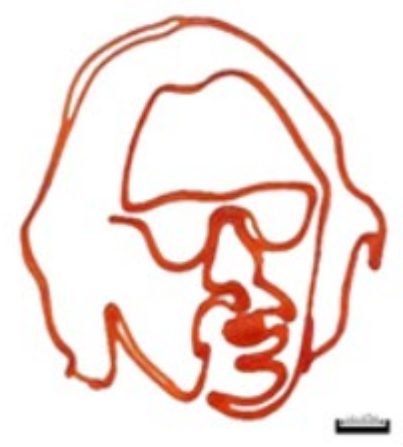

b)

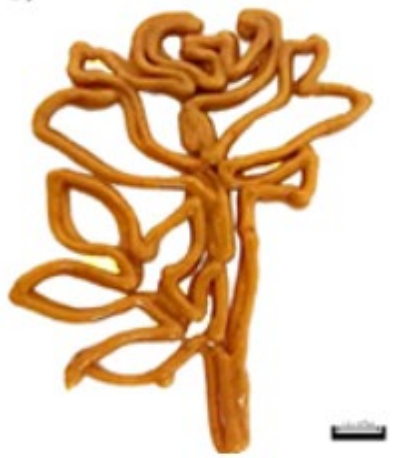

e)
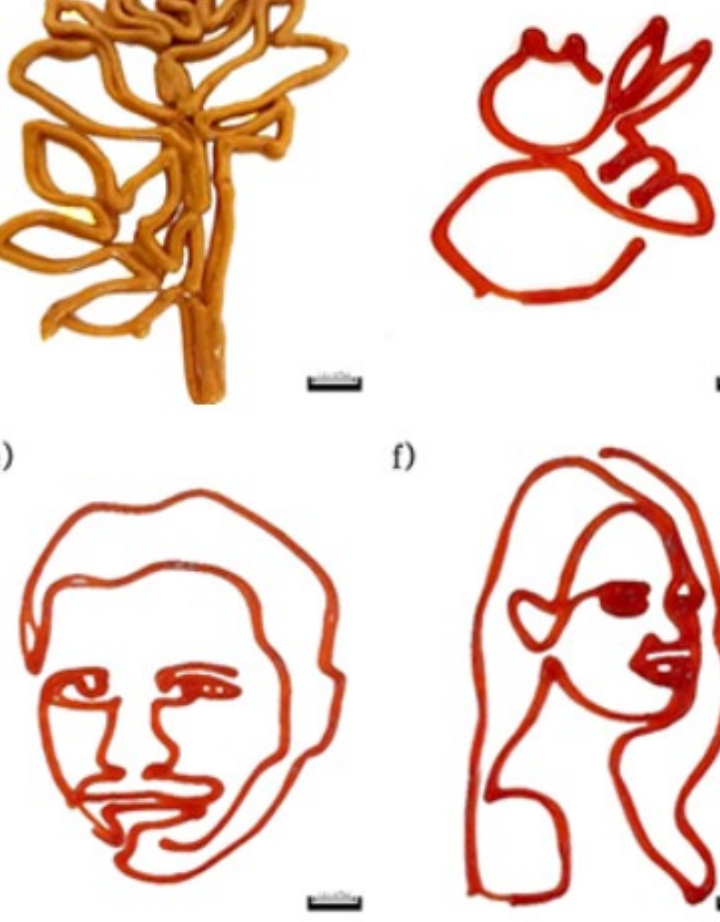

f)
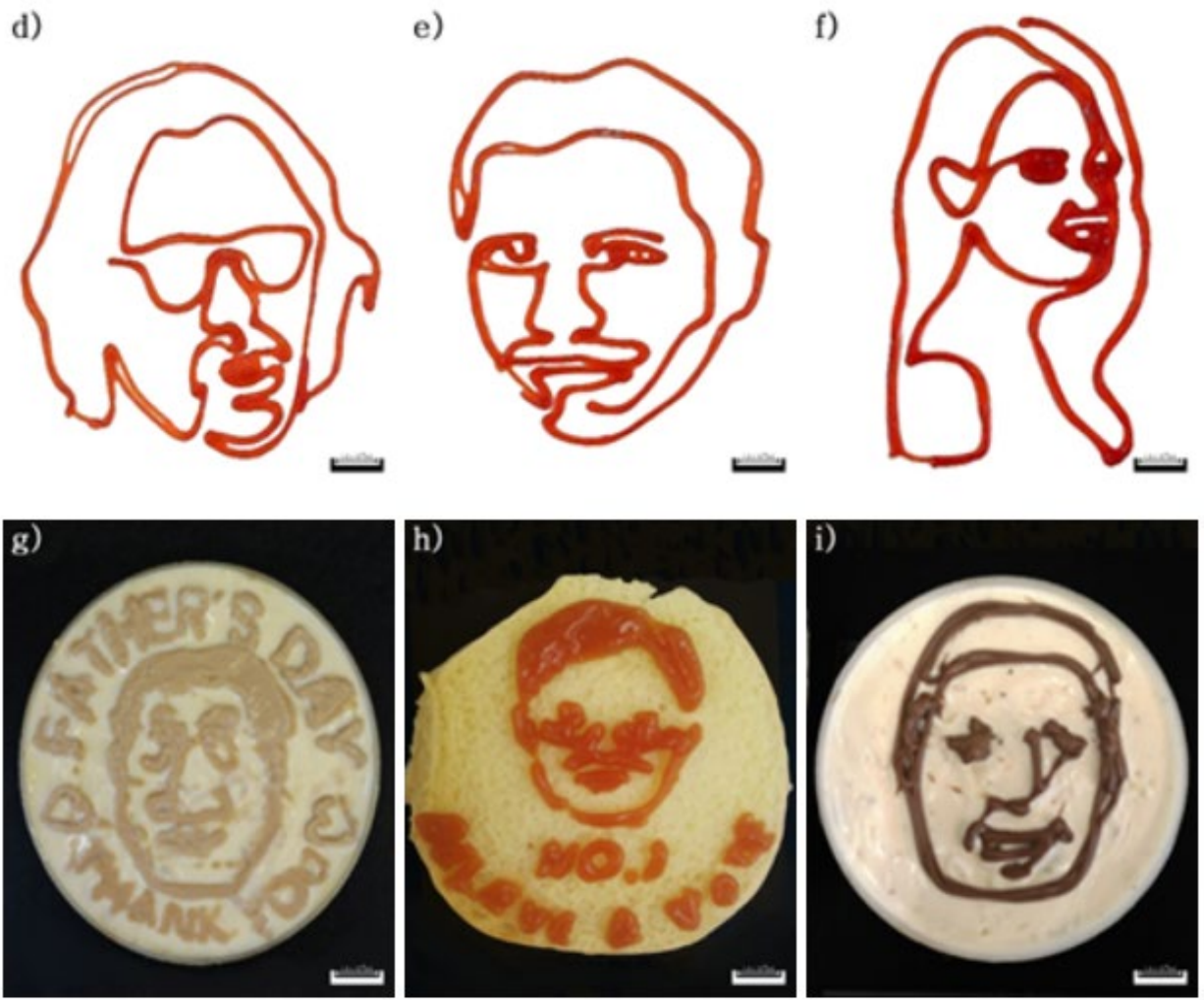

Figure 8. 3D printed designs using loT technology with various drug-loaded materials utilizing a tip-diameter of $1.66 \times 10^{-3} \mathrm{~m}$ and tip-length of $2.30 \times 10^{-2} \mathrm{~m}$. a)-b) peanut butter; c)-f) ketchup; g) yogurt; h) ketchup; i) chocolate pudding.

When loT technology is utilized, real-time data will be analyzed and used to identify the points where the shape can collapse, depending on the design or printing head speed. Then, the material can be discharged from the points where the shape will not collapse. This allows the patients to eat 3D printed food that will not subside in a short time.

By analyzing printing materials in real-time, each material can be stably printed in any design. This was possible by the optimization of the printing head speed, according to the type of material, viscosity, and number of bends in the design. As shown in Fig. 8, the prints of a design with various materials were stable every time without collapsing or spillage, even though each design was printed three times. It is noteworthy that a patient can choose any food material that is loaded with drugs and print the designs without any collapse in the figures or unappealing shapes.-The real-time analysis using IoT technology will allow the detection of any substance that was lost or overflowed during printing, giving specialists the capability of precisely determining the next prescription for the patient.

The potential for the IoT system to be applicable for patientspecific drugs is not only limited to real-time analysis of the design or printing status, but can also be utilized to monitor environmental conditions for material storage within the printer. This will allow the printer's systems to compensate for these factors and keep the material at optimal conditions for 
use when it is needed. As all medications have different prescription labels and handling guidelines, the medications that cannot be stored in large quantities outside of medical facilities or that need special containment will still need to be stored differently based on specialists' instructions or will need to be held at medical facilities.

The limitations that were observed for this experiment dealt with the constraints placed on the storage of the materials within the printers and with the security of individuals' data. The depletion of drugs or food material would need nurses or medical assistants to replenish the lost material in order for patients to continue receiving adequate care within their homes. In addition, the sensitive health data of patients' needs to be kept confidential and protected from potentially fraudulent activity. Future studies will present solutions to these limitations by examining procedures to sustain the levels of materials within remote 3D printers and by conducting research on methods to develop the security systems of the loT database.

\section{Conclusion}

The revolutionary patient-specific prescription has been successfully verified by converging 3D food printing technology with DDSs. Quantitative dosages of drugs were incorporated in the composition of various foods. Vitamin C and other food ingredients were precisely printed within $5 \%$ of the theoretical target during printing within five minutes. The prints could be created using various figures, regardless of the design or distance from where the print was ordered to where it was produced. The application of the loT-enabled 3D printer makes it possible to heal patients of their diseases given time and the correct nutritional plans for patients requiring an effective DDS. Consumers' daily use of the IoT system is a realistic implementation that will allow them to print their personalized meals. Ordinary users can customize any victual product with their choice of ingredients and shapes and have it produced in minutes. The customers can remove specific ingredients that they are allergic to or add the ones they prefer with accurate amounts. The loT technology will also monitor for changes in mood, appetite, health, and many other factors in real-time. This means both patients and day-to-day customers can video conference with a doctor who can diagnose their conditions and prescribe dosages of medication and food for them to intake. Exchange of messages or gifts using AM technology is applicable by representing special and unique shapes or images on the food. These claims have yet to be seen until now due to the emergence of IoT 3D printing, and the possible applications of this technology are copious. A better understanding of how this process would work and a step-by-step application from the medical field's perspective of DDSs will lead the way to real innovation.

\section{References}

1. Asiado T, Hippocrates Bio. https://www.ediblewildfood.com/bios/hippocrates.asp x. Accessed August 23, 2018.

2. Park KN. Controlled Drug Delivery Systems: Past Forward and Future Back. Journal of Controlled Release. 2014; 190: 3-8

3. Arnum PV, Drug Delivery: What May the Future Hold?. https://www.dcatvci.org/11-value-chain-insights/35drug-delivery-what-may-the-future-hold. Published August 10, 2016. Accessed August 20, 2018.

4. Newby JM, Seim I, Lysy M, et al. Technological strategies to estimate and control diffusive passage times through the mucus barrier in mucosal drug delivery. Advanced Drug Delivery Reviews. 2018; 124: 64-81

5. Kwon MH. Is the Ringer's Solution Cheaper than Water? The story of Ringer's Solution http://health.chosun.com/site/data/html_dir/2011/09/ 05/2011090500427.html. Published September 5, 2011. Accessed August 23, 2018.

6. Ringer Solution for Infusion. Electronic Medicines Compendium.

https://www.medicines.org.uk/emc/product/1865/smp c. Accessed August 24, 2018.

7. Kim NS. (2018). Foreign Patent No. PCTKR2017012766. Submitted.

8. Economidou SN, Lamprou DA, Douroumis D. 3D printing applications for transdermal drug delivery. International J. of Pharmaceutics. 2018; 544(2): 415-424.

9. Microfabricated Microneedles: A Novel Approach to Transdermal Drug Delivery. Journal of Pharmaceutical Sciences. 2000; 87(8): 922-925.

10. Hollander J, Hakala R, Suominen J, et al. 3D Printed UV Light Cured Polydimethylsiloxane Devices for Drug Delivery. International Journal of Pharmaceutics. 2018; 544(2): 433-442.

11. Kim NS. (2018). Foreign Patent No. D2019-0005. Submitted.

12. Acosta L. 3-D Printing Expands to School of Pharmacy. https://www.utep.edu/newsfeed/3-d-printing-expandsto-school-of-pharmacy.html. Published May 14, 2018. Accessed May 15, 2018.

13. Horvath G, Erdos G, Point Cloud Based Robot Cell Calibration. CIRP Annals. 2017; 66(1): 145-148

14. Ferdoush S, Li X. Wireless Sensor Network System Design Using Raspberry $\mathrm{Pi}$ and Arduino for Environmental Monitoring Applications. Procedia Computer Science. 2014; 34: 103-110.

15. Cruz MA, Rodrigues J, Sangaiah AK, et al. Performance evaluation of IoT middleware. Journal of Network and Computer Applications. 2018; 109: 53-65

16. Kim N, Eo J, Cho D. Optimization of Piston Type Extrusion (PTE) Techniques for 3D Printed Food. J. of Food Eng. 2018; 235(10): 41-49 
17. Tian J, Bryksa B, Yada R. Feeding the world into the future - food and nutrition security: the role of food science and technology. Frontiers in Life Science. 2016; 9(3): 155-166. https://doi.org/10.1080/21553769.2016.1174958

18. Hoffman J, Hwang S, Ortega A, et al. The Standardization of Printable Materials and Direct Writing Systems. J. Electron. Packag. 2013; 135(1), 011006.

19. Amza C, Zapciu A, Popescu D, Paste Extruder-Hardware Add-On for Desktop 3D Printers. Technologies. 2017; 5(3): 50-63.

20. Amert A, Oh D, Kim N. A simulation and experimental study on packing of nanoinks to attain better conductivity. J. of Appl. Phys. 2010; 108(10): 102806102806-5.

21. Hong S, Kim N. Synthesis of 3D Printable Cu-Ag Core-Shell Materials: Kinetics of CuO Film Removal. J. of Elec. Mat. 2015; 44(3): 823-830

22. Cesarano J, A Review of Robocasting Technology. MRS Proceedings. 1998; 543:133. https://doi.org/10.1557/PROC-542-133.

23. Kim NS. (2016). Korean Patent No. KR101834510B1. Seoul, Korea

24. Kim NS. (2018). Foreign Patent No. PCTKR2017008750. Submitted

25. Sanjay ST, Zhou W, Dou M, et al. Recent advances of controlled drug delivery using microfluidic platforms. Advanced Drug Delivery Reviews. 2018; 128: 3-28.

26. Mou X, Ali Z, Li S, et al. Application of magnetic nanoparticles in targeted drug delivery system. Journal of Nanoscience and Nanotechnology. 2015; 15(1): 54-62

27. Curci A, Natile G, Margiotta N. Drug targeting and delivery of platinum chemotherapeutics. Reference Module in Chemistry, Molecular Sciences and Chemical Engineering. 2018. https://doi.org/10.1016/B978-0-12409547-2.14282-9 